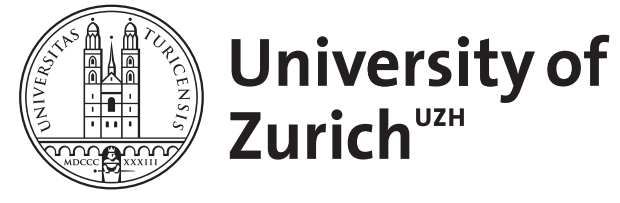

\title{
On simplification of combinatorial link Floer homology
}

Beliakova, A

Abstract: We define a new combinatorial complex computing the hat version of link Floer homology over $/ 2$, which turns out to be significantly smaller than the Manolescu-Ozsváth-Sarkar one.

DOI: https://doi.org/10.1142/S0218216510007796

Posted at the Zurich Open Repository and Archive, University of Zurich ZORA URL: https://doi.org/10.5167/uzh-38436

Journal Article

Accepted Version

Originally published at:

Beliakova, A (2010). On simplification of combinatorial link Floer homology. Journal of Knot Theory and Its Ramifications, 19(2):125-144.

DOI: https://doi.org/10.1142/S0218216510007796 


\title{
SIMPLIFICATION OF COMBINATORIAL LINK FLOER HOMOLOGY
}

\author{
ANNA BELIAKOVA
}

\begin{abstract}
We define a new combinatorial complex computing the hat version of link Floer homology over $\mathbb{Z} / 2 \mathbb{Z}$. Droz's computer program verifies that in many examples our complex is significantly smaller than the Manolescu-Ozsváth-Sarkar one.
\end{abstract}

\section{INTRODUCTION}

Knot Floer homology is a powerful knot invariant constructed by Ozsváth-Szabó [12] and Rasmussen [15]. In its basic form, the knot Floer homology $\widehat{\operatorname{HFK}}(K)$ of a knot $K \in S^{3}$ is a finite-dimensional bigraded vector space over $\mathbb{F}=\mathbb{Z} / 2 \mathbb{Z}$

$$
\widehat{\operatorname{HFK}}(K)=\bigoplus_{d \in \mathbb{Z}, i \in \mathbb{Z}} \widehat{\operatorname{HFK}}_{d}(K, i)
$$

where $d$ is the Maslov and $i$ is the Alexander grading. Its graded Euler characteristic

$$
\sum_{d, i}(-1)^{d} \operatorname{rank} \widehat{\operatorname{HFK}}_{d}(K, i) t^{i}=\Delta_{K}(t)
$$

is equal to the symmetrized Alexander polynomial $\Delta_{K}(t)$. The knot Floer homology enjoys the following symmetry extending that of the Alexander polynomial.

$$
\widehat{\operatorname{HFK}}_{d}(K, i)=\widehat{\mathrm{HFK}}_{d-2 i}(K,-i)
$$

By the result of Ozsváth-Szabó [11], the maximal Alexander grading $i$, such that $\widehat{\operatorname{HFK}}_{*}(K, i) \neq 0$ is the Seifert genus $g(K)$ of $K$. Moreover, Ghiggini showed for $g(K)=1$ [3] and $\mathrm{Yi} \mathrm{Ni}$ in general [8], that the knot is fibered if and only if $\operatorname{rank} \widehat{\operatorname{HFK}}_{*}(K, g(K))=1$. A concordance invariant bounding from below the slice genus of the knot can also be extracted from knot Floer homology [10]. For torus knots the bound is sharp, providing the new proof of Milnor conjecture. A purely combinatorial proof of the Milnor conjecture was given by Rasmussen in [16] by using Khovanov homology [4].

2000 Mathematics Subject Classification: 57R58 (primary), 57M27 (secondary)

Key words and phrases. Heegaard Floer homology, link invariants. 


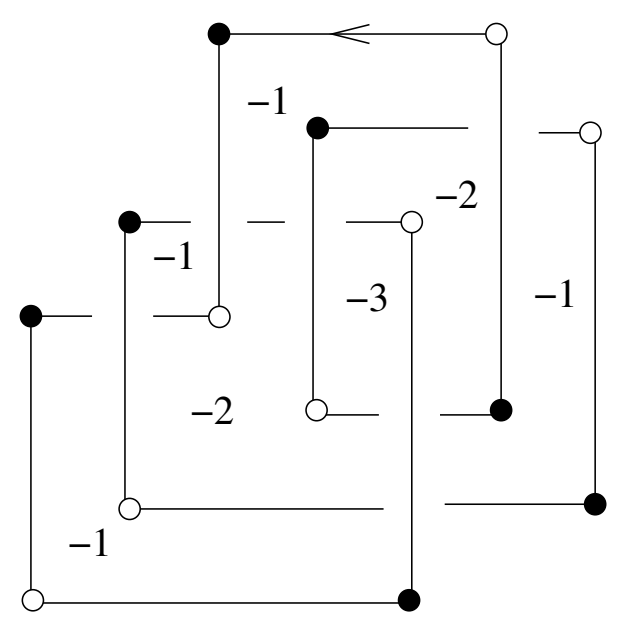

FiguRE 1. A rectangular diagram for $5_{2}$ knot. The number associated to a domain is minus the winding number for its points. The sets $\mathbb{X}$ and $\mathbb{O}$ consist of black and white points, respectively.

Knot Floer homology was extended to links in [14]. The first combinatorial construction of the link Floer homology was given in [6] over $\mathbb{F}$ and then in [7] over $\mathbb{Z}$. Both constructions use grid diagrams of links.

A grid diagram is a square grid on the plane with $n \times n$ squares. Each square is decorated either with an $X$, an $O$, or nothing. Moreover, every row and every column contains exactly one $X$ and one $O$. The number $n$ is called complexity of the diagram. Following [7], we denote the set of all $O$ 's and $X$ 's by $\mathbb{O}$ and $\mathbb{X}$, respectively.

Given a grid diagram, we construct an oriented, planar link projection by drawing horizontal segments from the $O$ 's to the $X$ 's in each row, and vertical segments from the $X$ 's to the $O$ 's in each column. We assume that at every intersection point the vertical segment overpasses the horizontal one. This produces a planar rectangular diagram $G$ for an oriented link $L$ in $S^{3}$. Any link in $S^{3}$ admits a rectangular diagram (see e.g. [2]). An example is shown in Figure 1.

In [6], [7] the grid lies on the torus, obtained by gluing the top most segment of the grid to the bottommost one and the leftmost segment to the right most one. In the torus, the horizontal and vertical segments of the grid become circles. The MOS complex is then generated by $n$-tuples of intersection points between horizontal and vertical circles, such that exactly one point belongs to each horizontal (or vertical) circle. Two generators $\mathbf{x}$ and $\mathbf{y}$ are connected by a differential if there exists a rectangle with vertices among $\mathbf{x}$ and $\mathbf{y}$ and edges lying alternatively on horizontal and vertical circles, which does not contain $X$ 's and $O$ 's and points among $\mathbf{x}$ and $\mathbf{y}$. 


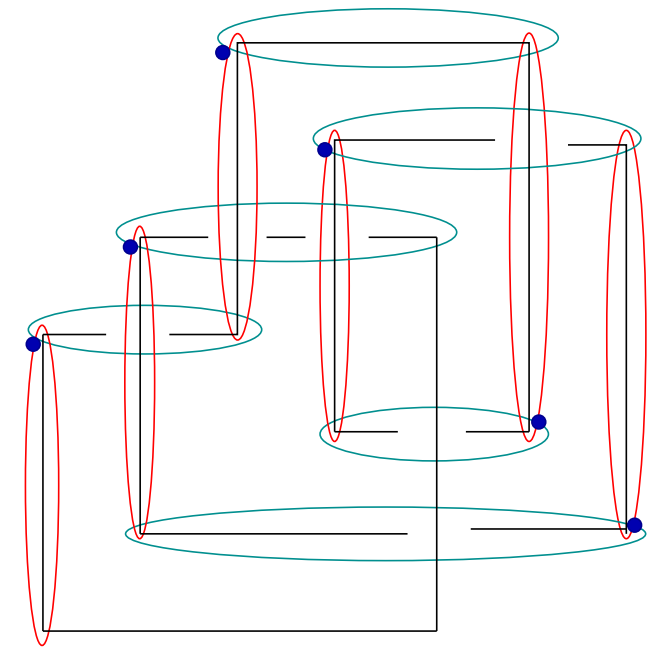

Figure 2. Admissible collection of ovals for $5_{2}$ knot. The dots show a generator in Alexander grading 1.

The Alexander grading is given by formula (3) below, and the Maslov grading by (4) plus one. The MOS complex has $n$ ! generators. This number greatly succeeds the rank of its homology. For the trefoil, for example, the number of generators is 120, while the rank of $\widehat{\operatorname{HFK}}\left(3_{1}\right)$ is 3 .

In this paper, we construct another combinatorial complex computing link Floer homology, which has significantly less generators. All knots with less than 6 crossings admit rectangular diagrams where all differentials in our complex are zero, and the rank of the homology group is equal to the number of generators.

Main results. Our construction also uses rectangular diagrams. Given an oriented link $L$ in $S^{3}$, let $G$ be its rectangular diagram in $\mathbb{R}^{2}$. Let us draw $2 n-2$ narrow ovals around all but one horizontal and all but one vertical segments of the rectangular diagram $G$. We denote by $\mathbf{S}$ the set of unordered $(n-1)$-tuples of intersection points between the horizontal and vertical ovals, such that exactly one point appears on each horizontal (or vertical) oval. We assume throughout this paper that the ovals intersect transversally. An example is shown in Figure 2.

Given $\mathbf{x}, \mathbf{y} \in \mathbf{S}$, there is an oriented closed curve $\gamma_{\mathbf{x}, \mathbf{y}}$ composed of arcs belonging to horizontal and vertical ovals, where each piece of a horizontal oval connects a point in $\mathbf{x}$ to a point in $\mathbf{y}$ (and hence each piece of the vertical one goes from a point in $\mathbf{y}$ to a point in $\mathbf{x}$ ). In $S^{2}=\mathbb{R}^{2} \cup \infty$, there exists a domain $D$ bounded by $\gamma_{\mathbf{x}, \mathbf{y}}$. Suppose that the orientation of $D$ is induced by the orientation of $S^{2}$. 
Let $D_{i}$ be the closures of the connected components of the complement of ovals in $S^{2}$. Then $D=\sum_{i} n_{i} D_{i}$. We say that $D$ connects two generators if for all $i, n_{i} \geq 0$. In particular, if $\gamma_{\mathbf{x}, \mathbf{y}}$ is oriented as the boundary of $D$, we denote $D$ by $D_{\mathbf{x}, \mathbf{y}}$ and say that it connects $\mathbf{x}$ to $\mathbf{y}$; otherwise, $D=D_{\mathbf{y}, \mathbf{x}}$ and it connects $\mathbf{y}$ to $\mathbf{x}$. The points $\mathbf{x}$ and $\mathbf{y}$ are called corners of the domain.

In [5], Lipshitz defined the Maslov index $M\left(D_{\mathbf{x}, \mathbf{y}}\right)$ of $D_{\mathbf{x}, \mathbf{y}}$ as follows. Let $k$ be the number of acute right-angled corners in $D_{\mathbf{x}, \mathbf{y}}$, and $l$ be the number of obtuse ones.

$$
M\left(D_{\mathbf{x}, \mathbf{y}}\right)=\chi\left(D_{\mathbf{x}, \mathbf{y}}\right)-k / 4+l / 4+n_{\mathbf{x}}+n_{\mathbf{y}},
$$

where $\chi(D)$ is the Euler characteristic of the domain $D$ considered as a 2-chain, and $n_{\mathbf{x}}=\sum_{x_{i} \in \mathbf{x}} n_{x_{i}}$ with $n_{x_{i}} \in\{0,1 / 4,1 / 2,3 / 4\}$ for an isolated (or common corner of $\mathbf{x}$ and $\mathbf{y}$ ), acute (i.e. $\pi / 2$-angled), straight (i.e. $\pi$-angled) or obtuse (i.e. $3 \pi / 2-$ angled) corner, respectively. For a composition of two domains $D_{\mathbf{x}, \mathbf{z}}=D_{\mathbf{x}, \mathbf{y}} \cup D_{\mathbf{y}, \mathbf{z}}$, we have $M\left(D_{\mathbf{x}, \mathbf{z}}\right)=M\left(D_{\mathbf{x}, \mathbf{y}}\right)+M\left(D_{\mathbf{y}, \mathbf{z}}\right)$.

We call a domain $D$ decomposable if it is a composition of Maslov index zero and one domains; otherwise, the domain is indecomposable.

A collection of ovals is called admissible, if all Maslov index one domains without $X$ 's and $O$ 's inside, connecting two elements of $\mathbf{S}$, are indecomposable. An example of the admissible collection is given by long ovals, where all ovals are as long as the $n \times n$ grid and the unbounded region contains a point among $X$ 's and $O$ 's (compare Figure 3).

A chain complex $(C(G), \partial)$ computing the hat version of link Floer homology of $L$ over $\mathbb{F}=\mathbb{Z} / 2 \mathbb{Z}$ is defined as follows. The generators are elements of $\mathbf{S}$ for an admissible collection of ovals. The differential of $\mathbf{x} \in \mathbf{S}$ is a sum over all $\mathbf{y} \in \mathbf{S}$, such that $\mathbf{x}$ can be connected to $\mathbf{y}$ by a Maslov index one domain, which does not contain $X$ 's and $O$ 's. Note that this domain is indecomposable by definition. If the ovals used to define $(C(G), \partial)$ are sufficiently short, this complex has less generators than the MOS complex (compare Section 3).

The gradings in $(C(G), \partial)$ can be constructed analogously to those in [6]. Suppose $\ell$ is the number of components of $L$. Let us introduce an Alexander-grading on $(C(G), \partial)$ as a function $A: \mathbf{S} \longrightarrow \mathbb{Z}^{\ell}$, defined as follows.

First, we define a function $a: \mathbf{S} \rightarrow \mathbb{Z}^{\ell}$. For a point $p$, the $i^{\text {th }}$ component of $a$ is minus the winding number of the projection of the $i^{\text {th }}$ component of the oriented link around $p$. In the grid diagram, we have $2 n$ distinguished squares containing $X$ 's or $O$ 's. Let $\left\{c_{i, j}\right\}, i \in\{1, \ldots, 2 n\}, j \in\{1, \ldots, 4\}$, be the vertices of these squares. Given $\mathbf{x} \in \mathbf{S}$, we set

$$
A(\mathbf{x})=\sum_{x \in \mathbf{x}} a(x)-\frac{1}{8}\left(\sum_{i, j} a\left(c_{i, j}\right)\right)-\left(\frac{n_{1}-1}{2}, \ldots, \frac{n_{\ell}-1}{2}\right),
$$




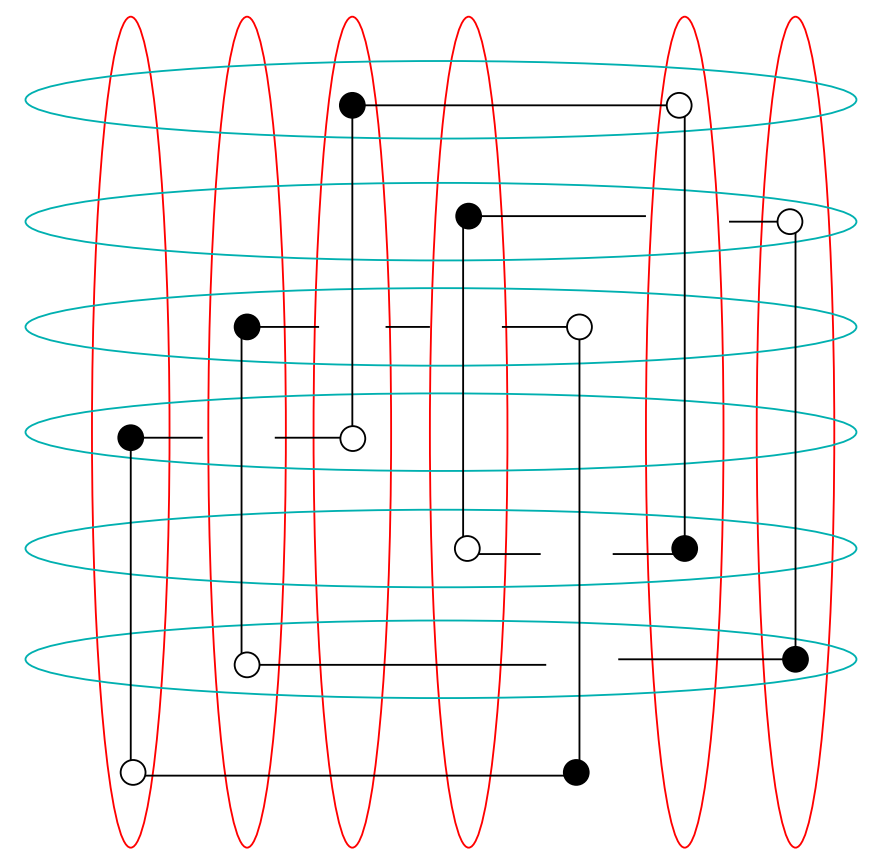

Figure 3. The complex $(C(G), \partial)$ with long ovals.

where here $n_{i}$ is the complexity of the $i^{\text {th }}$ component of $L$, i.e. the number of horizontal segments belonging to this component.

The homological or Maslov grading is given by a function $M: \mathbf{S} \rightarrow \mathbb{Z}$, which is defined as follows. Given two collections $A, B$ of finitely many points in the plane, let $\mathcal{I}(A, B)$ be the number of pairs $\left(a_{1}, a_{2}\right) \in A$ and $\left(b_{1}, b_{2}\right) \in B$ with $a_{1}<b_{1}$ and $a_{2}<b_{2}$. Define

$$
M(\mathbf{x})=\mathcal{I}(\mathbf{x}, \mathbf{x})-\mathcal{I}(\mathbf{x}, \mathbb{O})-\mathcal{I}(\mathbb{O}, \mathbf{x})+\mathcal{I}(\mathbb{O}, \mathbb{O})
$$

It is easy to see that the differential preserves the Alexander-grading and drops the Maslov-grading by one (compare Proposition 2.6 in [7]). Let $V_{i}$ be the two dimensional bigraded vector space over $\mathbb{F}$ spanned by one generator in Alexander and Maslov gradings zero and another one in Maslov grading -1 and Alexander grading minus the $i$-th basis vector.

Theorem 1. Suppose $G$ is a rectangular diagram of an oriented $\ell$ component link $L$, where the $i^{\text {th }}$ component of $L$ has complexity $n_{i}$. Then the homology $H_{*}(C(G), \partial)$ is equal to $\widehat{\operatorname{HFK}}(L) \otimes \bigotimes_{i=1}^{\ell} V_{i}^{n_{i}-1}$. 
We prove Theorem 1 by showing that $(C(G), \partial)$ is homotopy equivalent to the link Floer homology complex associated to a suitably chosen $2 n$-pointed Heegaard diagram of $S^{3}$ compatible with $L$. More precisely, given a rectangular diagram $G$ of complexity $n$, let $\left(C\left(S^{2}, \boldsymbol{\alpha}, \boldsymbol{\beta}, \mathbb{X}, \mathbb{O}\right), \partial\right)$ be the Ozsváth-Szabó complex with extra basepoints $\mathbb{X}$ and $\mathbb{O}$, defined in Section 1 . Here $\boldsymbol{\alpha}$ and $\boldsymbol{\beta}$ are $2(n-1)$ ovals in $S^{2}$ encircling all but one horizontal and vertical segments of $G$. The generators of this complex are $(n-1)$-tuples of intersection points between $\boldsymbol{\alpha}$ and $\boldsymbol{\beta}$ curves, and the differential counts Maslov index one domains without basepoints inside, which have holomorphic representatives.

Our main result is the following.

Theorem 2. The complex $\left(C\left(S^{2}, \boldsymbol{\alpha}, \boldsymbol{\beta}, \mathbb{X}, \mathbb{O}\right), \partial\right)$ is homotopy equivalent to a complex with the same generators, where any indecomposable Maslov index one domain connecting two generators without basepoints inside counts for the differential. A decomposable domain may or may not contribute to the differential.

The proof of Theorem 2 is organized as follows. By the deep results of Ozsváth and Szabó, $\left(C\left(S^{2}, \boldsymbol{\alpha}, \boldsymbol{\beta}, \mathbb{X}, \mathbb{O}\right), \partial\right)$ is homotopy equivalent to the long oval complex. Then we short the long ovals by means of a simple lemma from homological algebra keeping track of the differential. The methods developed in the proof allow to decide by purely algebraic means whether a domain counts or not. However, for decomposable domains the count depends on the order of shortening of ovals, which replace in some sense the choice of a complex structure in the analytic setting.

Remark. From the analytical point of view, Gromov compactness implies that the count of indecomposable domains does not depend on the complex structure, but it does not follow that this count is always $1(\bmod 2)$ in our case.

Our complex can easily be generalized to provide other variants of link Floer homology over $\mathbb{F}$. For the simplicity of exposition, we do not discuss these generalizations.

The paper is organized as follows. We recall the construction of link Floer homology in Section 1. Theorems 1 and 2 are proven in Section 2. In Section 3 we discuss computations made by Droz [1].

Acknowledgments. First of all, I wish to thank Stephen Bigelow for explaining to me his homological constructions of link invariants, which provided the original motivation for this project, and also for the regular conversations and helpful hints.

I would like to express my gratitude to Jean-Marie Droz for interesting discussions and, especially, for writing the program computing the homology of the complex constructed in this paper. 
I profited a lot from discussions with Stephan Wehrli. A special thank goes to Ciprian Manolescu for sharing his knowledge of Heegaard Floer homology and for his valuable suggestions after reading the preliminary version of this paper.

\section{Link Floer homology With MUltiple BASEPoints}

For the readers convenience, we review here the Ozsváth-Szabó construction of knot and link Floer homology, considering the case where the link meets the Heegaard surface in extra intersection points. Our exposition follows closely $[6$, Section $2]$.

Let $(\Sigma, \boldsymbol{\alpha}, \boldsymbol{\beta}, \mathbf{w}, \mathbf{z})$ be a Heegaard diagram for $S^{3}$, where $\Sigma$ is a surface of genus $g, k$ is some positive integer, $\boldsymbol{\alpha}=\left\{\alpha_{1}, \ldots, \alpha_{g+k-1}\right\}$ are pairwise disjoint, embedded curves in $\Sigma$ which span a half-dimensional subspace of $H_{1}(\Sigma ; \mathbb{Z}$ ) (and hence specify a handlebody $U_{\alpha}$ with boundary equal to $\left.\Sigma\right), \boldsymbol{\beta}=\left\{\beta_{1}, \ldots, \beta_{g+k-1}\right\}$ is another collection of attaching circles specifying $U_{\beta}$, and $\mathbf{w}=\left\{w_{1}, \ldots, w_{k}\right\}$ and $\mathbf{z}=\left\{z_{1}, \ldots, z_{k}\right\}$ are distinct marked points with

$$
\mathbf{w}, \mathbf{z} \subset \Sigma-\boldsymbol{\alpha}-\boldsymbol{\beta}
$$

Let $\left\{A_{i}\right\}_{i=1}^{k}$ resp. $\left\{B_{i}\right\}_{i=1}^{k}$ be the connected components of $\Sigma-\boldsymbol{\alpha}$ resp. $\Sigma-\boldsymbol{\beta}$.

We suppose that our basepoints are placed in such a manner that each component $A_{i}$ or $B_{i}$ contains exactly one basepoint among the $\mathbf{w}$ and exactly one basepoint among the $\mathbf{z}$. We can label our basepoints so that $A_{i}$ contains $z_{i}$ and $w_{i}$, and then $B_{i}$ contains $w_{i}$ and $z_{\nu(i)}$, for some permutation $\nu$ of $\{1, \ldots, k\}$.

In this case, the basepoints uniquely specify an oriented link $L$ in $S^{3}=U_{\alpha} \cup U_{\beta}$, by the following conventions. For each $i=1, \ldots, k$, let $\xi_{i}$ denote the arc in $A_{i}$ from $z_{i}$ to $w_{i}$ and let $\eta_{i}$ denote the arc in $B_{i}$ from $w_{i}$ to $z_{\nu(i)}$. Let $\widetilde{\xi}_{i} \subset U_{\alpha}$ be an arc obtained by pushing the interior of $\xi_{i}$ into $U_{\alpha}$, and $\widetilde{\eta}_{i}$ be the arc obtained by pushing the interior of $\eta_{i}$ into $U_{\beta}$. Now, we can let $L$ be the oriented link obtained as the union

$$
\bigcup_{i=1}^{k}\left(\widetilde{\xi}_{i}+\widetilde{\eta}_{i}\right)
$$

Definition 1.1. In the above case, we say that $(\Sigma, \boldsymbol{\alpha}, \boldsymbol{\beta}, \mathbf{w}, \mathbf{z})$ is a $2 k$-pointed Heegaard diagram compatible with the oriented link $L$ in $S^{3}$.

Let $\ell$ denote the number of components of $L$. Clearly, $k \geq \ell$. In the case where $k=$ $\ell$, these are the Heegaard diagrams used in the definition of link Floer homology [14], see also [12], [15]. In the case where $k>\ell$, these Heegaard diagrams can still be used to calculate link Floer homology, in a suitable sense. 
Definition 1.2. A periodic domain is a two-chain of the form

$$
P=\sum_{i=1}^{k}\left(a_{i} \cdot A_{i}+b_{i} \cdot B_{i}\right)
$$

such that $w_{i} \notin P$ for all $i$. A Heegaard diagram is said to be admissible if for every non-trivial periodic domain, the set $\left\{a_{i}, b_{i}\right\}$ contains some stricktly positive and negative numbers.

For simplicity, we consider now the case where $L$ is a knot. The case of links can be handled with a few minor notational changes in Subsection 1.1 below.

Let $(\Sigma, \boldsymbol{\alpha}, \boldsymbol{\beta}, \mathbf{w}, \mathbf{z})$ be a Heegaard diagram compatible with an oriented knot $K$. Let us consider the complex $C(\Sigma, \boldsymbol{\alpha}, \boldsymbol{\beta}, \mathbf{w}, \mathbf{z})$ generated over $\mathbb{F}$ by intersection points between tori $\mathbb{T}_{\alpha}=\alpha_{1} \times \cdots \times \alpha_{g+k-1}$ and $\mathbb{T}_{\beta}=\beta_{1} \times \cdots \times \beta_{g+k-1}$ in $\operatorname{Sym}^{g+k-1}(\Sigma)$ endowed with the differential

$$
\partial \mathbf{x}=\sum_{\mathbf{y} \in \mathbb{T}_{\alpha} \cap \mathbb{T}_{\beta}} \sum_{\substack{M(\phi)=1,\left\{\phi \in \pi_{2}(\mathbf{x}, \mathbf{y}) \mid\right.}} \#\left(\frac{\mathcal{M}(\phi)}{\mathbb{R}}\right) \cdot \mathbf{y}
$$

where $\pi_{2}(\mathbf{x}, \mathbf{y})$ denotes the space of homology classes of Whitney disks (domains) connecting $\mathbf{x}$ to $\mathbf{y}, M(\phi)$ is the Maslov index of $\phi, n_{p}(\phi)$ denotes the local multiplicity of $\phi$ at the reference point $p$ (i.e. the algebraic intersection number of $\phi$ with the subvariety $\left.\{p\} \times \operatorname{Sym}^{g+k-2}(\Sigma)\right), \mathcal{M}(\phi)$ is the moduli space of pseudo-holomorphic representatives of $\phi$, and \#() denotes a count modulo two. In the case when the Heegaard diagram is admissible, the sum in Equation (5) is finite. We refer to [13] for further details.

The relative Alexander grading of two intersection points $\mathbf{x}$ and $\mathbf{y}$ is defined by the formula

$$
A(\mathbf{x})-A(\mathbf{y})=\left(\sum_{i=1}^{k} n_{z_{i}}(\phi)\right)-\left(\sum_{i=1}^{k} n_{w_{i}}(\phi)\right) .
$$

The absolut $A$-grading can be fixed by requiring

$$
\sum_{\mathbf{x} \in \mathbb{T}_{\alpha} \cap \mathbb{T}_{\beta}} t^{A(\mathbf{x})} \equiv \Delta_{K}(t) \cdot\left(1-t^{-1}\right)^{n-1} \quad(\bmod 2),
$$

where $\Delta_{K}(t)$ is the symmetrized Alexander polynomial which could be made to work over $\mathbb{Z}$ by introducing signs.

Moreover, there is a relative Maslov grading, defined by

$$
M(\mathbf{x})-M(\mathbf{y})=M(\phi)-2 \sum_{i=1}^{k} n_{w_{i}}(\phi) .
$$


The relative Maslov grading can also be lifted to an absolute grading as explained in e.g. [6].

For this complex, the function $A$ defines an Alexander grading which is preserved by the differential. The following proposition shows how to extract the usual knot Floer homology from the above variants using multiple basepoints. The proof is given in [14], compare also [6].

Proposition 1.1. Let $(\Sigma, \boldsymbol{\alpha}, \boldsymbol{\beta}, \mathbf{w}, \mathbf{z})$ be a $2 k$-pointed admissible Heegaard diagram compatible with a knot $K$. Then, we have an identification

$$
H_{*}(C(\Sigma, \boldsymbol{\alpha}, \boldsymbol{\beta}, \mathbf{w}, \mathbf{z}), \partial) \cong \widehat{\operatorname{HFK}}(K) \otimes V^{\otimes(k-1)},
$$

where $V$ is the two-dimensional vector space spanned by two generators, one in bigrading $(-1,-1)$, another in bigrading $(0,0)$.

1.1. Modifications for links. Recall that knot Floer homology has a generalization to the case of oriented links $L$. For an $\ell$ component oriented link $L$ in $S^{3}$, this takes the form of a multi-graded theory

$$
\widehat{\operatorname{HFK}}(L)=\bigoplus_{d \in \mathbb{Z}, h \in \mathbb{H}} \widehat{\operatorname{HFK}}_{d}(L, h)
$$

where $\mathbb{H} \cong H_{1}\left(S^{3}-L\right) \cong \mathbb{Z}^{\ell}$, with the latter isomorphism induced by an ordering of the link components. We sketch now the changes to be made to the above discussion to define link Floer homology for Heegaard diagrams with extra basepoints.

Suppose now that $(\Sigma, \boldsymbol{\alpha}, \boldsymbol{\beta}, \mathbf{w}, \mathbf{z})$ is a Heegaard diagram compatible with an oriented link $L$ in the sense of Definition 1.1.

Let us label the basepoints keeping track of which link component they belong to. Specifically, suppose $L$ is a link with $\ell$ components, and for $i=1, \ldots, \ell$, we choose $n_{i}$ basepoints to lie on the $i^{\text {th }}$ component. Letting $S$ be the index set of pairs $(i, j)$ with $i=1, \ldots, \ell$ and $j=1, \ldots, n_{i}$. We now have basepoints $\left\{z_{i, j}\right\}_{(i, j) \in S}$ and $\left\{w_{i, j}\right\}_{(i, j) \in S}$.

We can now form the chain complex $C(\Sigma, \boldsymbol{\alpha}, \boldsymbol{\beta}, \mathbf{w}, \mathbf{z})$ over $\mathbb{F}$ analogous to the version before, generated by intersection points of $\mathbb{T}_{\alpha} \cap \mathbb{T}_{\beta}$. This complex has a relative Maslov grading as before. It also has an Alexander grading which in this case is an $\ell$-tuple of integers,

$$
A: \mathbb{T}_{\alpha} \cap \mathbb{T}_{\beta} \longrightarrow \mathbb{Z}^{\ell},
$$

determined up to an overall additive constant by the formula

$$
A(\mathbf{x})-A(\mathbf{y})=\left(\sum_{j=1}^{n_{1}}\left(n_{z_{1, j}}(\phi)-n_{w_{1, j}}(\phi)\right), \ldots, \sum_{j=1}^{n_{\ell}}\left(n_{z_{\ell, j}}(\phi)-n_{w_{\ell, j}}(\phi)\right)\right) .
$$

The indeterminacy in this case can be fixed with the help of Proposition 1.2. 
The differential drops Maslov grading by one and preserves the Alexander multigrading, and hence the homology groups $H_{*}(C(\Sigma, \boldsymbol{\alpha}, \boldsymbol{\beta}, \mathbf{w}, \mathbf{z}))$ inherit a Maslov grading and an Alexander multi-grading.

Proposition 1.2. Let $(\Sigma, \boldsymbol{\alpha}, \boldsymbol{\beta}, \mathbf{w}, \mathbf{z})$ be a $2 k$-pointed admissible Heegaard diagram compatible with an oriented link $L$, with $n_{i}$ pairs of basepoints corresponding to the $i^{\text {th }}$ component of $L$. Then, there are multi-graded identifications

$$
H_{*}(C(\Sigma, \boldsymbol{\alpha}, \boldsymbol{\beta}, \mathbf{w}, \mathbf{z}), \partial) \cong \widehat{\operatorname{HFK}}(L) \otimes \bigotimes_{i=1}^{\ell} V_{i}^{\otimes\left(n_{i}-1\right)},
$$

where $V_{i}$ is the two-dimensional vector space spanned by one generator in Maslov and Alexander gradings zero, and another in Maslov grading -1 and Alexander grading corresponding to minus the $i^{\text {th }}$ basis vector.

By the result of [14], the bigraded groups $\widehat{\mathrm{HFK}}(L)$ are link invariants. In particular, they do not change if

- the complex structure on $\Sigma$ is varied;

- the $\boldsymbol{\alpha}$ - and $\boldsymbol{\beta}$-curves are moved by isotopies (in the complement of the basepoints);

- the $\boldsymbol{\alpha}$ - and $\boldsymbol{\beta}$-curves are moved by handle-slides (in the complement of the basepoints);

- the Heegaard diagram is stabilized.

1.2. MOS complex. It was shown in [6], that for a link of complexity $n$ the MOS complex, described in Introduction, coincides with the complex $(C(\Sigma, \boldsymbol{\alpha}, \boldsymbol{\beta}, \mathbf{w}, \mathbf{z}), \partial)$, where $\Sigma$ is a torus, $\boldsymbol{\alpha}$ are $n$ parallel meridians on $\Sigma, \boldsymbol{\beta}$ are $n$ parallel longitudes (both providing $n \times n$ grid on the torus), and where the basepoints $\mathbf{w}$ and $\mathbf{z}$ are identified with $\mathbb{X}$ and $\mathbb{O}$. Hence, by Proposition 1.2 the homology of this complex is equal to $\widehat{\operatorname{HFK}}(L) \otimes \bigotimes_{i=1}^{\ell} V_{i}^{\otimes\left(n_{i}-1\right)}$

\section{ProOFS}

2.1. Shortening of ovals. Suppose $G$ be a rectangular diagram of complexity $n$ for an oriented link $L$. Let $(C, \partial)$ be the complex $\left(C\left(S^{2}, \boldsymbol{\alpha}, \boldsymbol{\beta}, \mathbb{X}, \mathbb{O}\right), \partial\right)$, where $\boldsymbol{\alpha}$ and $\boldsymbol{\beta}$ are $2(n-1)$ ovals encircling all but one horizontal and vertical segments of $G$, respectively. It is easy to check that any periodic domain in this case has positive and negative coefficients. The differential $\partial$ is given by counting of all Maslov index one domains connecting two generators, which do not contain $X$ 's and $O$ 's and admit holomorphic representatives. 


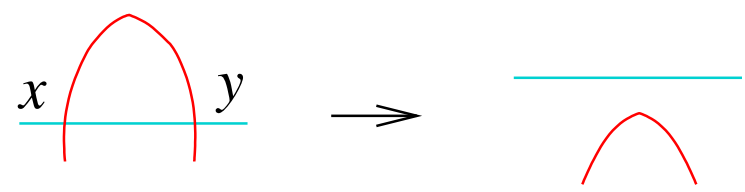

\section{FiguRE 4. Removing of a bigon without basepoints inside.}

The next lemma allows to "short" an oval by removing any bigon without basepoints inside (see Figure 4).

Lemma 2.1. Assume that $\boldsymbol{\alpha}$ and $\boldsymbol{\beta}$ curves form a bigon with corners $x$ and $y$ without basepoints inside. Then the complex $(C, \partial)$ is homotopy equivalent to the complex $\left(C^{\prime}, \partial^{\prime}\right)$ whose set of generators $\mathbf{S}\left(C^{\prime}\right)$ is obtained from $\mathbf{S}(C)$ by removing all generators containing $x$ or $y$.

Proof. Let us define the homotopy equivalence explicitly.

Let $I: \mathbf{S}\left(C^{\prime}\right) \rightarrow \mathbf{S}(C)$ and $P: \mathbf{S}(C) \rightarrow \mathbf{S}\left(C^{\prime}\right)$ be the obvious inclusion and projection. For any $\mathbf{x} \in \mathbf{S}(C)$, let $h(\mathbf{x})$ be zero if $y \notin \mathbf{x}$, otherwise $h(\mathbf{x})$ is obtained from $\mathbf{x}$ by replacing $y$ by $x$. We define $F: \mathbf{S}(C) \rightarrow \mathbf{S}\left(C^{\prime}\right)$ and $G: \mathbf{S}\left(C^{\prime}\right) \rightarrow \mathbf{S}(C)$ as follows.

$$
F=P(\operatorname{Id}+\partial h) \quad G=(\operatorname{Id}+h \partial) I
$$

Here Id is the identity map. Then $G F-\mathrm{Id}=\partial h+h \partial$, i.e. $G F$ is homotopic to the identity on $C$. On the other hand, $F G$ is the identity map on $C^{\prime}$.

Let us endow the complex $C^{\prime}$ with the differential $\partial^{\prime}=F \partial G$. It is easy to check that $\partial^{\prime 2}=0$ and that $F$ and $G$ are chain maps. Indeed, $\partial^{\prime} F=F \partial I P$ and $I P \partial G=G \partial^{\prime}$, or $\partial^{\prime} F=F \partial$ and $\partial G=G \partial^{\prime}$ over $\mathbb{F}$.

Let us describe the new differential $\partial^{\prime}=P(\partial+\partial h \partial) I$ in more details. Assume $\mathbf{y} \in \partial \mathbf{x}$ and $\mathbf{x}, \mathbf{y}$ do not contain the corners of the bigon $x$ and $y$. For all such $\mathbf{x}$ and $\mathbf{y}, \partial$ and $\partial^{\prime}$ coincide, i.e. we have $\mathbf{y} \in \partial^{\prime} \mathbf{x}$. In addition, $\partial^{\prime}$ is nonzero in the following case. Assume $\mathbf{a}, \mathbf{b} \in \mathbf{S}(C)$ do not contain $x$ and $y$, and $\mathbf{x}, \mathbf{y} \in \mathbf{S}(C)$ contain these points and $h(\mathbf{y})=\mathbf{x}$. If, in addition, $\mathbf{y} \in \partial \mathbf{a}$, and $\mathbf{b} \in \partial \mathbf{x}$, then $\mathbf{b} \in \partial^{\prime} \mathbf{a}$.

2.2. Proof of Theorem 2. As before, given a grid diagram $G$, we denote by $(C, \partial)$ the complex $\left(C\left(S^{2}, \boldsymbol{\alpha}, \boldsymbol{\beta}, \mathbb{X}, \mathbb{O}\right), \partial\right)$.

Let $(F, \partial)$ be the complex $\left(C\left(S^{2}, \boldsymbol{\alpha}^{\prime}, \boldsymbol{\beta}^{\prime}, \mathbb{X}, \mathbb{O}\right), \partial\right)$ defined with long ovals. By the result of Ozsváth and Szabó, $(F, \partial)$ is homotopy equivalent to $(C, \partial)$. Note that over $\mathbb{F}$ quasi-isomorphism implies homotopy equivalence. Let $\left(C^{\prime}, \partial^{\prime}\right)$ be obtained from $(F, \partial)$ by applying Lemma 2.1 several times until $\left(C^{\prime}, \partial^{\prime}\right)$ has the same set of generators as $(C, \partial)$. We prove the claim by showing that in $\left(C^{\prime}, \partial^{\prime}\right)$ any indecomposable Maslov index one domain, connecting two generators and not containing 


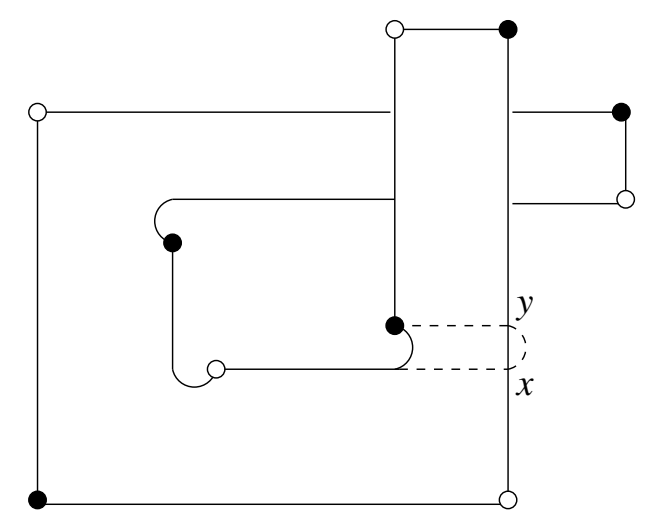

FIGURE 5. Immersed polygon realizing a differential from black to white points. The prolongated oval is shown by a dashed line.

$X$ 's and $O$ 's, counts for the differential. The proof is by induction on the number of boundary components $c$ in the domain.

Let $c=1$. Any bigon counts by the Riemann mapping theorem. Assume that any indecomposable immersed polygon with $2 n$ corners counts. Suppose that our complex has an immersed polygon $D$ with $2(n+1)$ corners without basepoints inside, connecting two generators. Then by prolongating one oval in the complement of basepoints, $D$ can be split into two polygons, say $P$ and $P^{\prime}$, with less than $2(n+1)$ corners (compare Figure 5). Let us denote by $C^{\prime \prime}$ the complex obtained after prolongating the oval. By Lemma 2.1, $\left(C^{\prime}, \partial^{\prime}\right)$ and $\left(C^{\prime \prime}, \partial^{\prime \prime}\right)$ are homotopy equivalent. By assumption, $P$ and $P^{\prime}$ count for the differential in $C^{\prime \prime}$. Moreover, their corners (after removing the corners of the bigon) define $\mathbf{a}$ and $\mathbf{b}$ as in Proof of Lemma 2.1, such that $\mathbf{b} \in \partial^{\prime} \mathbf{a}$ is given by counting $D=D_{\mathbf{a}, \mathbf{b}}$.

Assume that for $c=n-1$, the claim holds. Suppose the complex $C^{\prime}$ has an indecomposable Maslov index one domain $D$ with $c=n$ boundary components and without basepoints inside. Examples with $c=2$ are drawn in Figures 6, 7.

Let us stretch one oval in $D$, in such a way that $D$ gives rise to a domain $D^{\prime}$ with $c=n-1$ boundary components, i.e. $D^{\prime}$ has Maslov index two. Let $x$ and $y$ be the corners of the bigon, obtained after stretching. The stretched oval connects $y$ to some boundary component, say $B$. By Proposition 2.3 below, $y$ can also be connected to $B$ by a unique path inside $D$. Hence, $D^{\prime}$ can be represented as a union of two domains connecting some generators. The unique path connecting $y$ to $B$ leaves any boundary component along a cut. Moreover, if the path goes along the boundary of a component without obtuse corners, then it also meets a component with two obtuse corners (compare Proof of Theorem 2.3). Therefore, $D^{\prime}$ is a union 


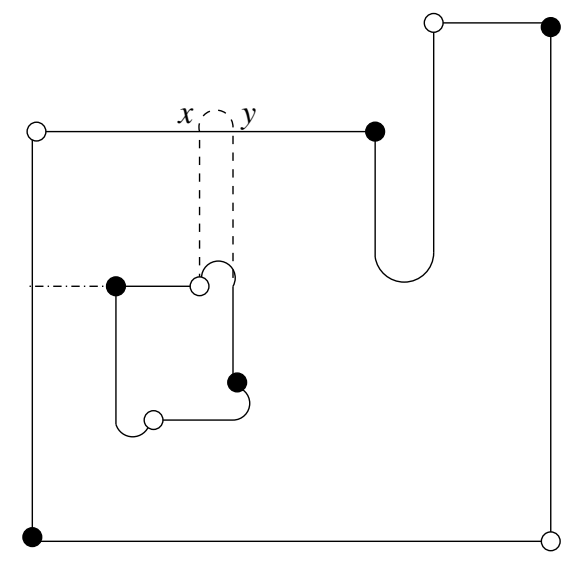

Figure 6. An indecomposable annulus realizing a differential from black to white points. The prolongated oval is shown by a dashed line.

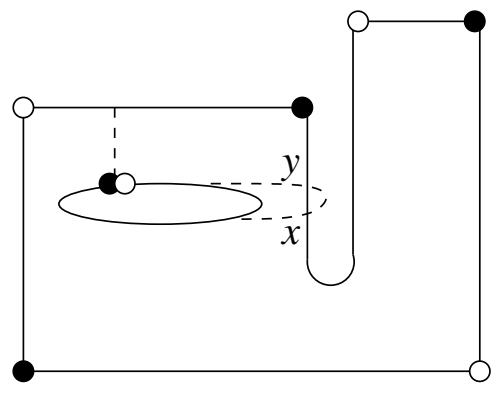

FiguRE 7. An indecomposable domain with straight corners.

of two Maslov index one domains. Removing $x$ and $y$ and also the end points of the cuts from the corners of these domains, we obtain $\mathbf{a}$ and $\mathbf{b}$ as in Proof of Lemma 2.1. Therefore, domain $D$ counts for the differential $\partial^{\prime}$.

2.3. Proof of Theorem 1. By Proposition 1.2, the homology of the complex $(C, \partial)$ is given by the following formula.

$$
H_{*}(C, \partial) \cong \widehat{\operatorname{HFK}}(L) \otimes \bigotimes_{i=1}^{\ell} V_{i}^{\otimes\left(n_{i}-1\right)},
$$

By Theorem 2, $(C, \partial)$ is homotopy equivalent to a complex with the same generators, where all indecomposable Maslov index one domains count for the differential. In the case where there are no decomposable domains, the last complex coincides with $(C(G), \partial)$. 
a)

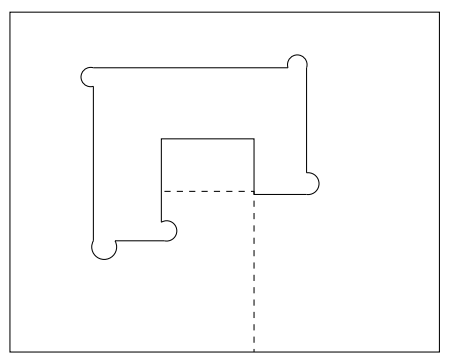

b)

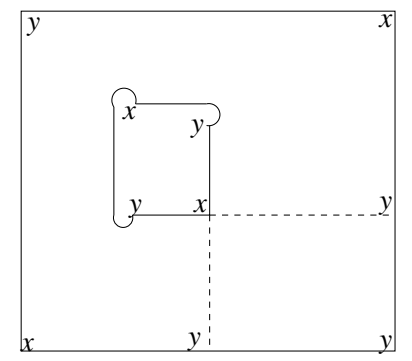

Figure 8. Case $b=0, c=2$. a) Decomposable domain. The cuts are shown by dashed lines. $b$ ) Indecomposable domain. The corners from $\mathbf{x}$ are marked by $x$ and the points from $\tilde{\mathbf{y}}$ by $y$.

2.4. Structure of indecomposable domains. Let us denote by $D_{\mathbf{x}, \mathbf{y}}$ a Maslov index one domain connecting $\mathbf{x}$ to $\mathbf{y}$. Let $c$ be the number of boundary components of $D_{\mathbf{x}, \mathbf{y}}$. We call a boundary component $b a d$, if it has no obtuse corners. Let $b$ be the number of bad components in the domain. A path in $D_{\mathbf{x}, \mathbf{y}}$ starting at an obtuse or straight corner and following a horizontal or vertical oval until the boundary of $D_{\mathbf{x}, \mathbf{y}}$ will be called a cut. There are two cuts at any obtuse corner and one at any straight corner.

Definition 2.2. A boundary component $C_{1} \subset \partial D_{\mathbf{x}, \mathbf{y}}$ is called $y$-connected to another component $C_{2} \subset \partial D_{\mathbf{x}, \mathbf{y}}$ if for any point $y \in C_{1}$ disjoint from the corners, there exists a unique path starting at $y$ and ending in $C_{2}$, such that

1) the path goes along cuts or $\partial D_{\mathbf{x}, \mathbf{y}}$, where the segments of horizontal and vertical ovals alternate along the path;

2) the corners of the path (i.e. intersection points of horizontal and vertical segments) come alternatively from $\mathbf{x}$ and $\tilde{\mathbf{y}}$, where $\tilde{\mathbf{y}}$ contains $\mathbf{y}$ and intersection points of cuts with $\partial D_{\mathbf{x}, \mathbf{y}}$;

3) the first corner belongs to $\mathbf{x}$.

Proposition 2.3. If $D_{\mathbf{x}, \mathbf{y}}$ is indecomposable Maslov index one domain, then any two connected components of its boundary are $y$-connected.

Proof. Let us first assume that $D_{\mathbf{x}, \mathbf{y}}$ has no straight corners. In this case, we prove the claim by induction on $c$ and $b$. Assume $b=0, c=2$. If one of the cuts goes from the inner boundary component to itself, the domain is decomposable (see Figure 8 $(a))$. If it is not the case, then an easy check verifies the claim (compare Figure 8 (b)).

Assume the claim holds for $b=0, c=n-1$. Let as add an $n$-th good component $A$ to the domain. Then if there are no cuts meeting $A$, we are done, since two cuts from 


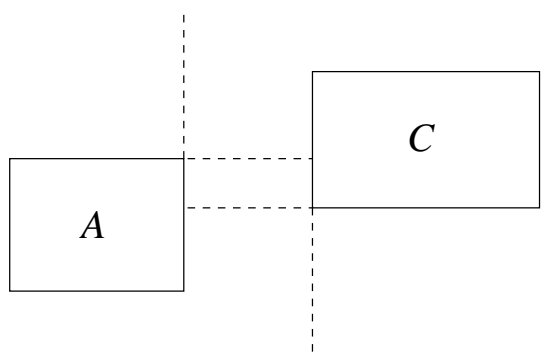

Figure 9. Case $b=0, c=n$. Decomposable domain. All corners without cuts are assumed to be acute.
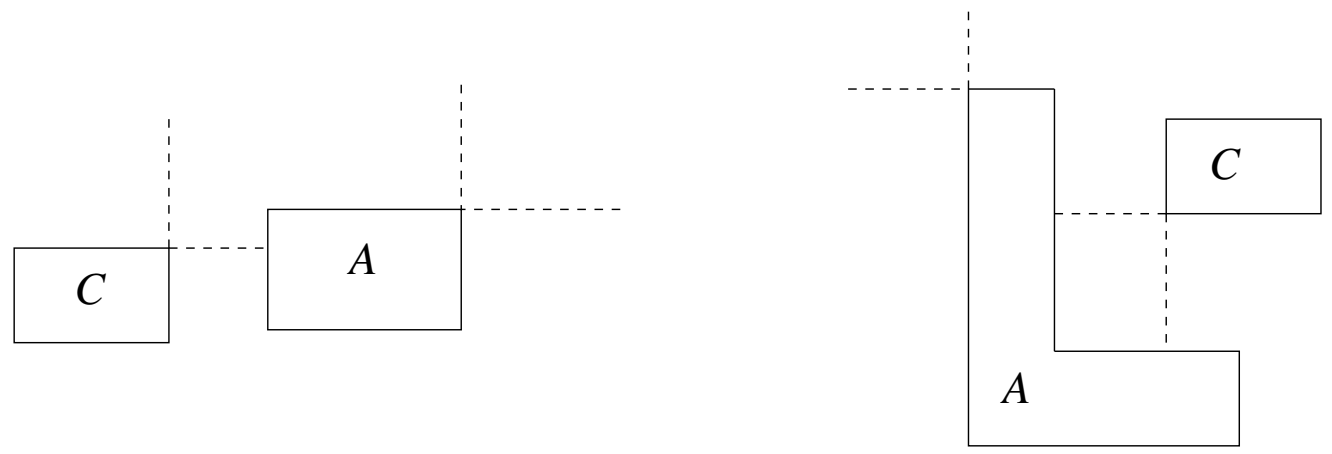

Figure 10. $C$ is connected to $A$ by one or two cuts. All corners without cuts are assumed to be acute.

the obtuse corner $y$-connect $A$ to any other component by the induction hypothesis. If $C \subset \partial D_{\mathbf{x}, \mathbf{y}}$ is connected to $A$ by a cut, then no cut from $A$ meets $C$, otherwise the domain is decomposable (compare Figure 9). It remains two possibilities shown in Figure 10. It is easy to check that in both cases $C$ and $A$ are $y$-connected to all other components. For this, it is sufficient to find a required path for two choices of $y$ (before and after one corner) on each component. An example is shown in Figure 11. Note that a particular form of the domain does not matter for the argument.

Assume $b=1, c=n$. Let us denote by $B$ the bad component. Then, since $M\left(D_{\mathbf{x}, \mathbf{y}}\right)=1$, there exists a component $E \subset \partial D_{\mathbf{x}, \mathbf{y}}$ with two obtuse corners. If two cuts from different obtuse corners connect $E$ to $B$ or to $\partial D_{\mathbf{x}, \mathbf{y}}-B$, then the domain is decomposable. Indeed, in these cases, $E$ is connected to $B$ or to the other components (which are $y$-connected by the induction hypothesis) by two paths, which allow to split the domain into Maslov index one and zero domains. Therefore, two cuts from one obtuse corner should connect $E$ to $B$ and two cuts from the other obtuse corner to $\partial D_{\mathbf{x}, \mathbf{y}}-B$. If a cut connecting $E$ to $B$ goes through other boundary 

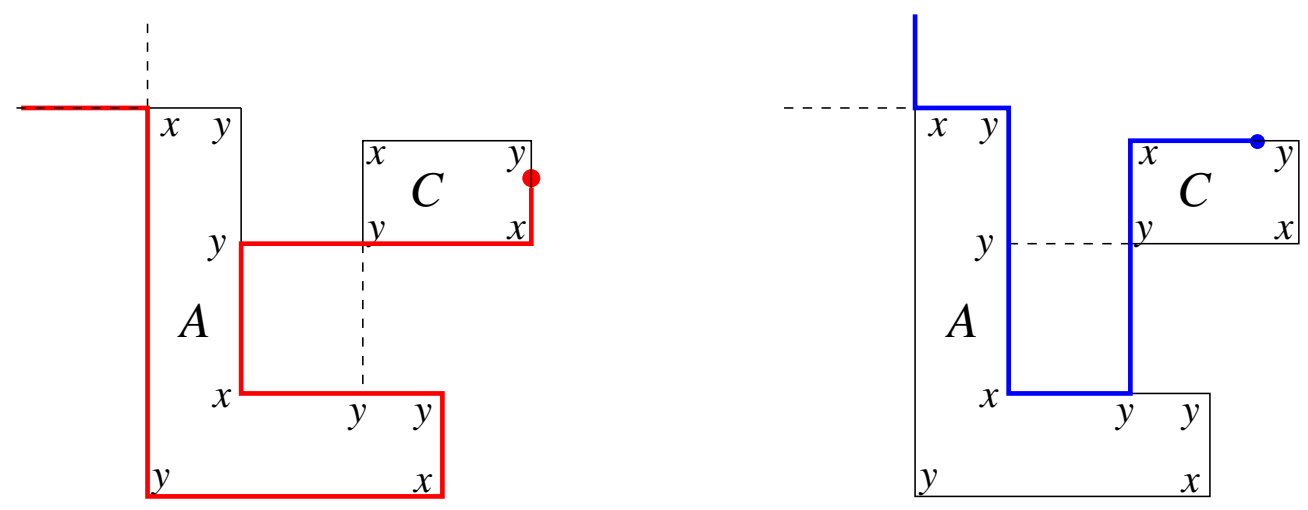

Figure 11. $C$ is $y$-connected to all other components. The two choices of $y$ are shown by red and blue dots. The connected paths have the corresponding colors.

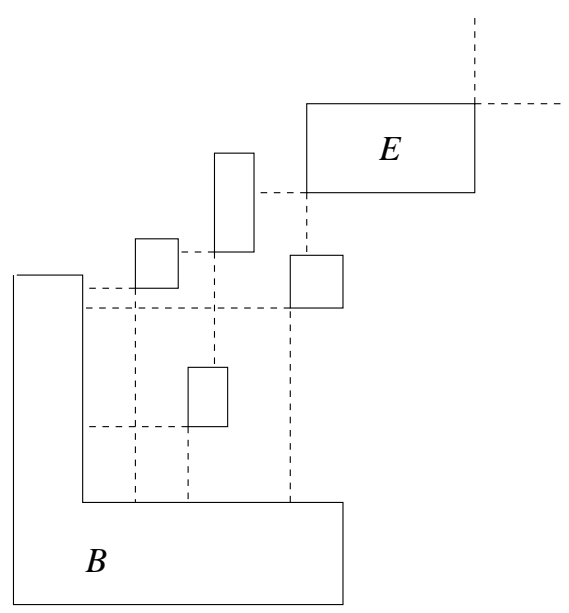

Figure 12. Case $b=1, c=n$. All drawn components are $y$ connected. All corners without cuts are assumed to be acute.

components, then no of them can be connected to $\partial D_{\mathbf{x}, \mathbf{y}}-B$ or to each other; otherwise the domain is decomposable by the same arguments as before. Hence, the situation looks like in Figure 12, where all components are $y$-connected.

If there are components connected to $B$ by one or two cuts, they are also $y-$ connected to the remaining components, since $B$ is $y$-connected to them.

In the case $b=k, c=n$, we assume that the domain is obtained from $b=k-1$, $c=n-1$ domain by adding one bad component $B$ and making one corner obtuse. All other components are $y$-connected to each other by the induction hypothesis. 

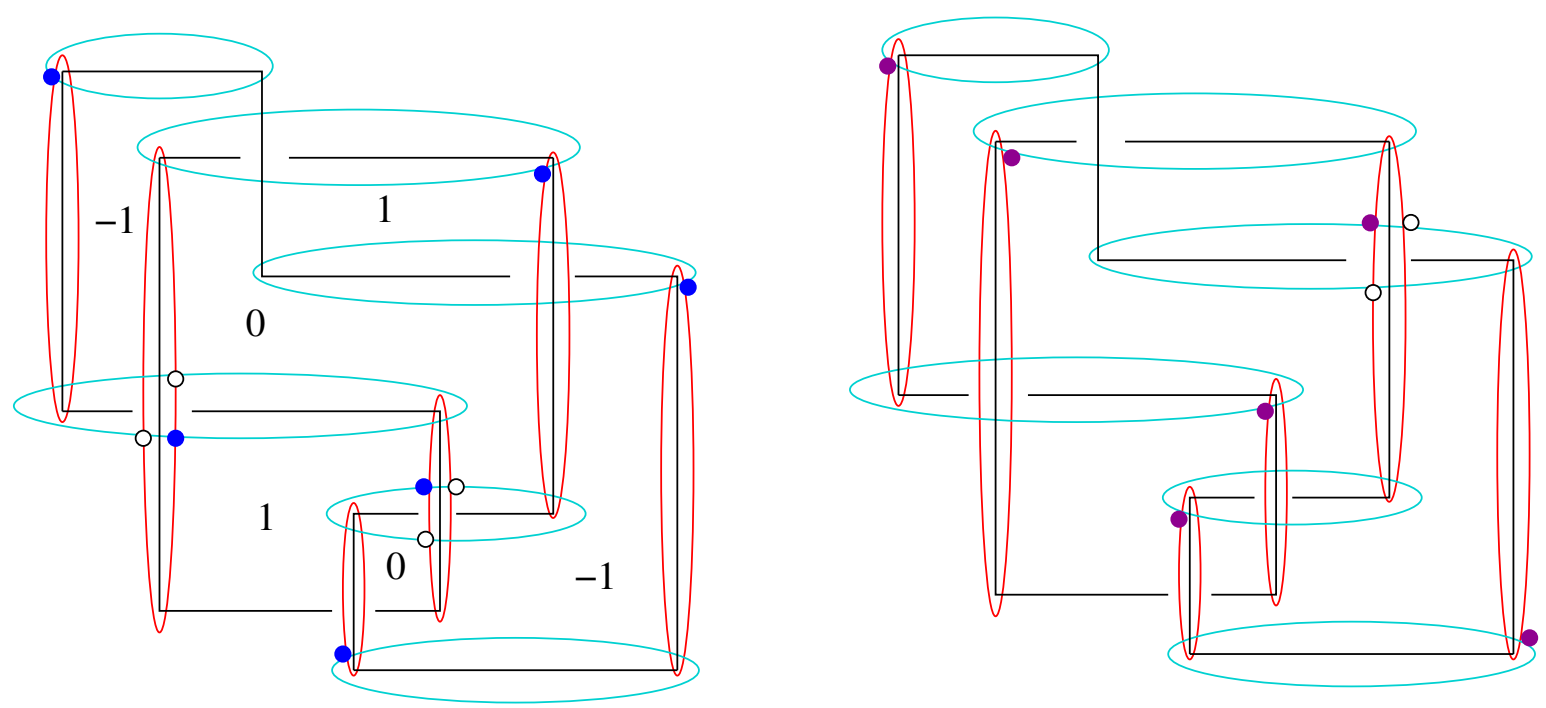

Figure 13. A complex for $5_{2}$ knot. The colored dots show generators in the maximal Alexander grading equal to 1. A number assigned to a region is minus the winding number for its points.

The new obtuse corner should be connected to $B$ by two cuts as in Figure 12 . The rest is similar to the previous case.

It remains to consider the case where $D_{\mathbf{x}, \mathbf{y}}$ has straight corners. In this case, one of the boundary components is an oval with a common corner of two generators. Let us call such a component special. An example is shown in Figure 7. Let us first assume that we have only one special component. Then the cut from the straight corner $y$-connects this component to any other one, by the previous argument. The obvious induction completes the proof.

\section{Computations}

3.1. $5_{2}$ knot. Figure 13 shows a rectangular diagram for $5_{2}$ knot of complexity $n=7$ obtained from the original diagram in Figure 1 by cyclic permutations (compare [2]). An advantage of this diagram is that there are no regions counted for the differential.

The Alexander grading of a generator is given by the formula $A(\mathbf{x})=\sum_{x \in \mathbf{x}} a(x)-$ 2. The maximal Alexander grading is equal to one. There are two generators in this grading shown by colored dots in Figure 13. Both of them have Maslov grading 2.

The homology of our complex is $\widehat{\operatorname{HFK}}\left(5_{2}\right) \otimes V^{6}$. Hence in Alexander grading zero, we have 12 additional generators coming from the multiplication with $V$. Note that our complex has 15 generators in Alexander grading zero. Indeed, 12 of them can be 
obtained by moving one point of a generator in Alexander grading one to the other side of the oval. In three cases, depicted by white dots there are two possibilities to move a point. This gives 3 additional generators. Note that all movings drop Maslov index by one. We deduce that $\widehat{\operatorname{HFK}}\left(5_{2}, 0\right)$ has rank three. To compute $\widehat{\mathrm{HFK}}$ in the negative Alexander gradings we use the symmetry (1).

Finally, we derive that $\widehat{\operatorname{HFK}}\left(5_{2}\right)$ has rank two in the Alexander-Maslov bigrading $(1,2)$, rank three in $(0,1)$, and rank two in the bigrading $(-1,0)$. To compare, the Alexander polynomial is $\Delta_{5_{2}}(t)=2\left(t+t^{-1}\right)-3$. The knot $5_{2}$ is not fibered and its Seifert genus is one.

3.2. Further examples. Droz wrote a computer program calculating the homology of our complex [1]. As a byproduct, his program generates rectangular diagrams of knots and links and allows to change them by Cromwell-Dynnikov moves.

According to his computations, all knots until 13 crossings and some more complicated knots admit rectangular diagrams, where maximally short ovals are admissible. In all these cases, the number of generators in our complex is significantly smaller than that in the MOS complex.

Indeed, for all knots admitting rectangular diagrams of complexity 10, the number of generators in the MOS complex is $10 !=3^{\prime} 628^{\prime} 800$. Our complex has on average about 50'000 generators among them about 1'000 in the positive Alexander gradings.

The knot $12 n 2000$ admits a rectangular diagram of complexity 12 , where 12 ! = $479^{\prime} 001^{\prime} 600$. Our complex has $1^{\prime} 411^{\prime} 072$ generators with $16^{\prime} 065$ in the positive Alexander gradings.

\section{REFERENCES}

[1] J.-M. Droz. Python program at www.math.unizh.ch/assistenten/jdroz

[2] I. Dynnikov. Arc-presentations of links: monotonic simplification. Fund. Math., 190:29-76, 2006.

[3] P. Ghiggini. Knot Floer homology detects genus-one fibred links. math.GT/0603445, 2006.

[4] M. Khovanov. A categorification of the Jones polynomial. Duke Math. J., 101(3):359-426, 2000.

[5] R. Lipshitz. A cylindrical reformulation of Heegaard Floer homology. math.SG/0502404, 2005.

[6] C. Manolescu, P. Ozsvàth, S. Sarkar. A combinatorial description of knot Floer homology. math.GT/0607691

[7] C. Manolescu, P. Ozsvàth, Z. Szabó, D. Thurston. On combinatorial link Floer homology. math.GT/0610559

[8] Y. Ni. Knot Floer homology detects fibred knots. math.GT/0607156.

[9] P. S. Ozsváth and Z. Szabó. Heegaard Floer homology and alternating knots. Geom. Topol., $7: 225-254,2003$. 
[10] P. S. Ozsváth and Z. Szabó. Knot Floer homology and the four-ball genus. Geom. Topol., 7:615-639, 2003.

[11] P. S. Ozsváth and Z. Szabó. Holomorphic disks and genus bounds. Geom. Topol., 8:311-334, 2004.

[12] P. S. Ozsváth and Z. Szabó. Holomorphic disks and knot invariants. Adv. Math., 186(1):58116, 2004.

[13] P. S. Ozsváth and Z. Szabó. Holomorphic disks and topological invariants for closed threemanifolds. Ann. of Math. (2), 159(3):1027-1158, 2004.

[14] P. S. Ozsváth and Z. Szabó. Holomorphic disks, link invariants, and the multi-variable Alexander polynomial. math.GT/0512286, 2005.

[15] J. A. Rasmussen. Floer homology and knot complements. PhD thesis, Harvard University, 2003.

[16] J. A. Rasmussen. Khovanov homology and the slice genus. math.GT/0402131, 2004.

Institut FÜr Mathematik, Universität ZÜRICh, Winterthurerstrasse 190, CH-8057 ZÜRICH, SwitZERLAND

E-mail address: anna@math.unizh.ch 